\title{
Transparent Conducting Electrodes for Efficient Organic Photovoltaic Devices under Indoor Light Conditions: A Brief Review
}

\author{
Received 5 October, 2020; revised 23 November, 2020; accepted 21 December, 2020
}

\author{
Ji Soo Goo ${ }^{a, *}$ \\ a Avionics R\&D center, Hanwha Systems, Seongnam 13524, Republic of Korea
}

*Corresponding author E-mail: jisoo.goo@hanwha.com

\begin{abstract}
Indoor organic photovoltaics (OPVs) are drawing increasing attention due to their considerable potential for supplying energy to low-power devices under indoor lighting, and exhibit advantages such as changeable optical absorption and cost-effective fabrication. When designing indoor OPVs, the photovoltaic materials, electrodes, and interlayers should be developed considering the indoor lighting conditions, which differ from the outdoor conditions. In this review, design principles such as adoption of high shunt resistance and enhancement of the light absorption for the transparent conducting electrodes (TCEs) of indoor OPVs are suggested. Subsequently, an overview of the recent developments in the TCEs of OPVs under indoor lighting conditions is presented. Furthermore, the future implications of this research topic is discussed.
\end{abstract}

Keywords: Organic photovoltaic devices, Indoor lighting conditions, Transparent conducting electrode, Shunt resistance, Equivalent-circuit model

\section{Introduction}

Recently, there has been an increasing interest in indoor energy harvesting systems with increasing use of small-scale electronic devices in residential applications, wearable electronic devices, and wireless sensor networks for Internet-of-Things (IoT) devices [1-3]. The replacement of chemical batteries and commercial electricity by the energy harvested from light, mechanical vibration, and heat have been extensively studied [4-6]. Among these energy sources, light and heat are preferred based of their performance in indoor applications. However, to obtain sufficient energy from a heat source, large thermal differences are necessary. Therefore, photovoltaic technology that converts light energy into electricity is more appropriate than others.

Organic photovoltaic (OPV) technology can be promising for indoor energy harvesting owing to its cost-effective fabrication, and optical properties such as high absorption coefficient and changeable optical bandgap. In addition, the advantages of OPVs including mechanical flexibility, light weight, environment friendly characteristics, and aesthetic advantages owing to the availability of various colors render them the preferred indoor energy harvester compared to the other sources [7-13]. In addition, recent reports indicate that OPVs have better photovoltaic performance compared to other photovoltaic technologies such as Si solar cells and copper-indium-gallium (di) selenide (CIGS) solar cells, as indoor light sources [14].

However, indoor and outdoor lighting conditions differ significantly in terms of the intensity and irradiation spectrum. The intensities of typical indoor lighting sources such as light emitting diode (LED), halogen light, and fluorescent light are only $0.05-1 \%$ that of the outdoor light. Moreover, the irradiation spectra of the indoor light differ from the solar spectrum, as depicted in Fig. 1. Therefore, different approaches are required while designing indoor OPVs [15].

In this study, we review the recent progress in the development of

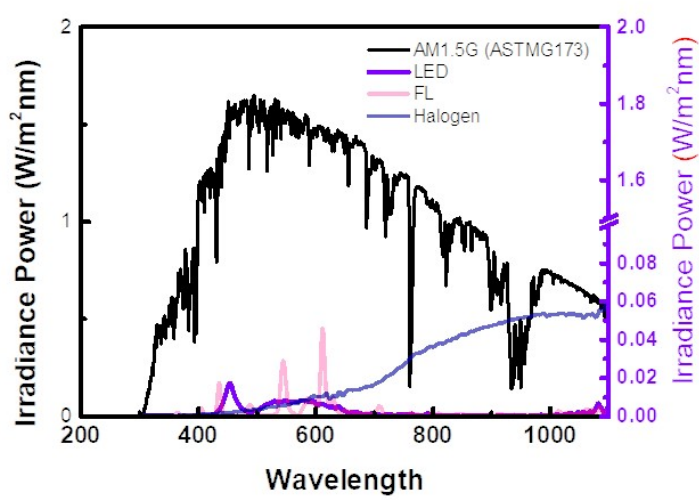

Figure 1. Spectra of light sources (AM 1.5G, 500-lx LED, 500-lx FL, and 500-lx Halogen).

indoor OPVs, focusing on transparent conducting electrodes (TCEs) of the devices. Furthermore, after comparing the device performances under outdoor and indoor lighting conditions for different electrodes, key strategies for electrode selection to realize efficient indoor OPVs are discussed. Finally, the future implications on this research topic are discussed.

\section{Theoretical background}

The typical OPV structures are displayed in Figs. 2(a) and 2(b). OPVs mainly comprise a donor:acceptor bulk-heterojunction (BHJ) sandwiched between two electrodes and two transport layers. When light passes through TCE, absorption occurs at $\mathrm{BHJ}$ and excitons are 
(a)

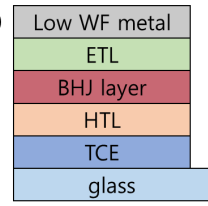

(b)
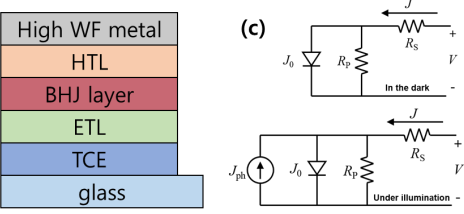

Figure 2. Typical OPV structure: (a) conventional geometry, (b) inverted geometry, and (c) equivalent circuit model

generated. The electrons and holes are separated from the excitons and transferred to the cathode and anode through the electron transport layer (ETL) and hole transport layer generating the required voltage and current [16-18].

To maximize OPV performance under indoor lighting conditions, approaches different from 1-sun illumination are required. For discussing the electrical properties, an organic solar cell can be approximated by an equivalent circuit [Fig. 2(c)] such as a current source (corresponding to the current density under illumination $J_{p h}$ ), an ideal diode with reverse saturation current (current density in the dark at reverse bias $\left.J_{0}\right)$, and two resistances $\left(R_{S}\right.$ and $\left.R_{P}\right)$. Based on this equivalent circuit, the open circuit voltage $\left(V_{O C}\right)$ can be expressed using the Shockley equation:

$$
\begin{gathered}
V_{O C}=\frac{k T}{e}\left\{1+\frac{J_{p h}}{J_{0}}\left(1-\frac{V_{O C}}{J_{p h} R_{P} A}\right)\right\} \approx \frac{k T}{e} \ln \left\{1+\frac{J_{p h}}{J_{0}}\right\} \\
J_{S C}=-\frac{1}{1+R_{S} / R_{P}}\left[J_{p h}-J_{0}\left\{\exp \left(\frac{\left|J_{S C}\right| R_{S} A}{n k T / e}\right)-1\right\}\right] \approx-J_{p h}
\end{gathered}
$$

The series resistance $\left(R_{S}\right)$, which includes the electrode resistance, material resistance, and contact resistance between the electrodes and the semiconductors, needs to be minimized for realizing the best solar cell performance. The shunt resistance $\left(R_{P}\right)$, which must be maximized, is related to the loss of carriers through possible leakage paths by the pinholes in the device and recombination at an interface. Therefore, OPVs with higher $\mathrm{R}_{P}$ present lower leakage currents.

According to Eqs. (1) and (2), among the components of the circuit model, $R_{P}$ plays an important role due to the reduced current density under indoor lighting conditions. The photocurrent density $\left(J_{p h}\right)$ generated in OPV is proportional to the light intensity. General indoor light sources such as LED, fluorescent, and Halhgen provide intensities ranging from of $200-1000 \mathrm{~lx}$, whereas the AM1.5G provides approximately $120000 \mathrm{~lx}$. Accordingly, the OPV photocurrent density $\left(J_{p h}\right)$ under indoor lighting conditions is in the range of $\sim \mu \mathrm{Acm}^{-2}$, which is within one order of scale of the leakage current. In devices with low $R_{P}$, the leakage current causes a drop in $V_{O C}$ and fill factor (FF), resulting in low-power conversion efficiency. As the light intensity decreases, the bias point and currents $\left(J_{p h}\right)$ passing through the solar cell also decrease, and the equivalent resistance of the solar cell begins to approach $R_{P}$. If these two resistances are similar, the fraction of the total current flowing through $R_{P}$ increases, increasing the fractional power loss. Recently, Steim et al. demonstrated the effect of $R_{P}$ and $R_{S}$ on the indoor and outdoor performances of OPVs through simulation and measurement of the performance of P3HT:PC ${ }_{61} \mathrm{BM}$ BHJ devices at various $R_{S}$ and $R_{P}$ values [19]. The devices exhibited up to $7 \%$ PCE under a 1000-lx lamp with a high $R_{P}\left(85 \mathrm{k} \Omega \cdot \mathrm{cm}^{2}\right)$ and $2.7 \%$ PCE under 1-sun illumination. Simulation of the relationship between $V_{O C}$ and the light intensity indicated that high $R_{\mathrm{P}}$ resulted in a low $V_{O C}$, on decreasing the light intensity. To obtain the full $V_{O C}$ of P3HT:PC ${ }_{61} \mathrm{BM}$ devices under low-light conditions, an $R_{P}$ of approximately $100 \mathrm{k} \Omega \cdot \mathrm{cm}^{2}$ was required. Moreover, Zhou et al. established the role of $R_{P}$ in the device performance and demonstrated that a sufficiently large $R_{P}$ value was crucial for the device under low-light intensity [20]. In summary, high $R_{P}$ as well as low $R_{S}$ are critical for achieving high PCE under 1-sun illumination; however, high $R_{P}$ is a crucial factor that determines the device properties under low-light conditions, regardless of $R_{S}$.

\section{Results and discussion}

As mentioned above, the electrode sheet resistances related to the $R_{S}$ value have negligible influence on the OPV performance in indoor conditions (low-light intensity), when $R_{\mathrm{P}}$ of the device is sufficiently large. Based on this property, various electrodes that are unsuitable for outdoor conditions due to low conductivity can be applied to indoor OPVs.

In 2019, Goo et al. used the $\mathrm{ZnO}$ layer as the cathode in inverted OPVs [21]. ZnO electrodes of various thicknesses were fabricated through the ALD process to obtain defect-free films. P3HT: ICBA BHJ devices with 200-nm-thick-ZnO electrodes offered 9.5\% PCE under 500-lx LED conditions, whereas they exhibited poor performance $(0.4 \%)$ under 1-sun illumination, as shown in Fig. 3. In addition, devices with $200-\mathrm{nm}$-thick- $\mathrm{ZnO}$ electrodes showed better performances than those with ITO electrodes. As the thickness of the $\mathrm{ZnO}$ layer also had significant effect on the device performance, at least 200-nm thickness was required to avoid current leakage. These results clearly indicate that other factors including a large $R_{P}$ value are more critical for realizing efficient indoor OPVs, compared to the TCE $R_{S}$ value.

The conductivity of PEDOT: PSS electrodes is insufficient for application in outdoor solar cells. However, Saeed et al. applied these electrodes in indoor OPVs and demonstrated their stability in flexible devices. Devices with polymer electrodes exhibited $11 \%$ PCE under 500-lx LED conditions, whereas the reference devices (PEN/ITO) exhibited only $8.8 \%$ PCE [22].

Interface engineering can be one of the strategies for indoor OPVs because the charge transport layer is directly related to the device $R_{S}$ and $R_{P}$, which significantly affect device performance. As the thickness of the PEIE layer is proportional to the concentration of the PEIE solution, various PEIE concentrations have been adopted to optimize conventional OPV geometry. Lechêne et al. established that the PEIE thickness had no effect on the device performance under outdoor lighting, whereas it had significant effect under indoor lighting [23]. Devices with considerable PEIE thickness exhibit high $R_{P}$, due to its insu- (a)

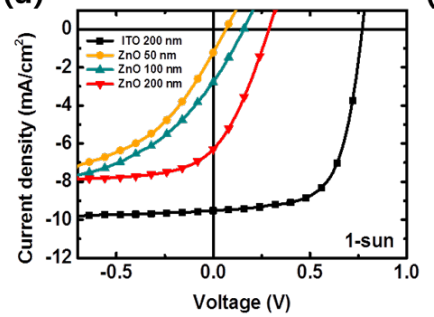

(b)

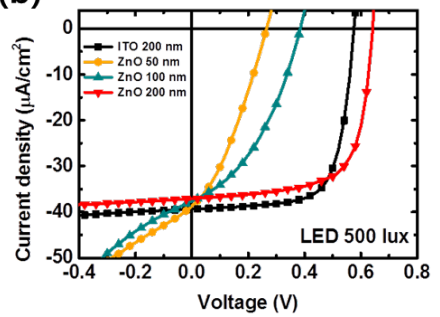

(c)

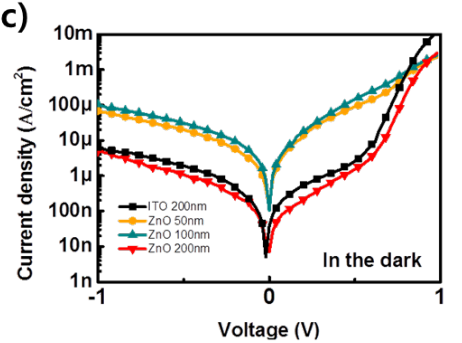

Figure 3. Characteristics of device with undoped ZnO electrodes under (a) 1-sun, (b) 500-lx LED, and (c) dark conditions. (Goo [21] 2018 Copyright Royal Society of Chemistry). 

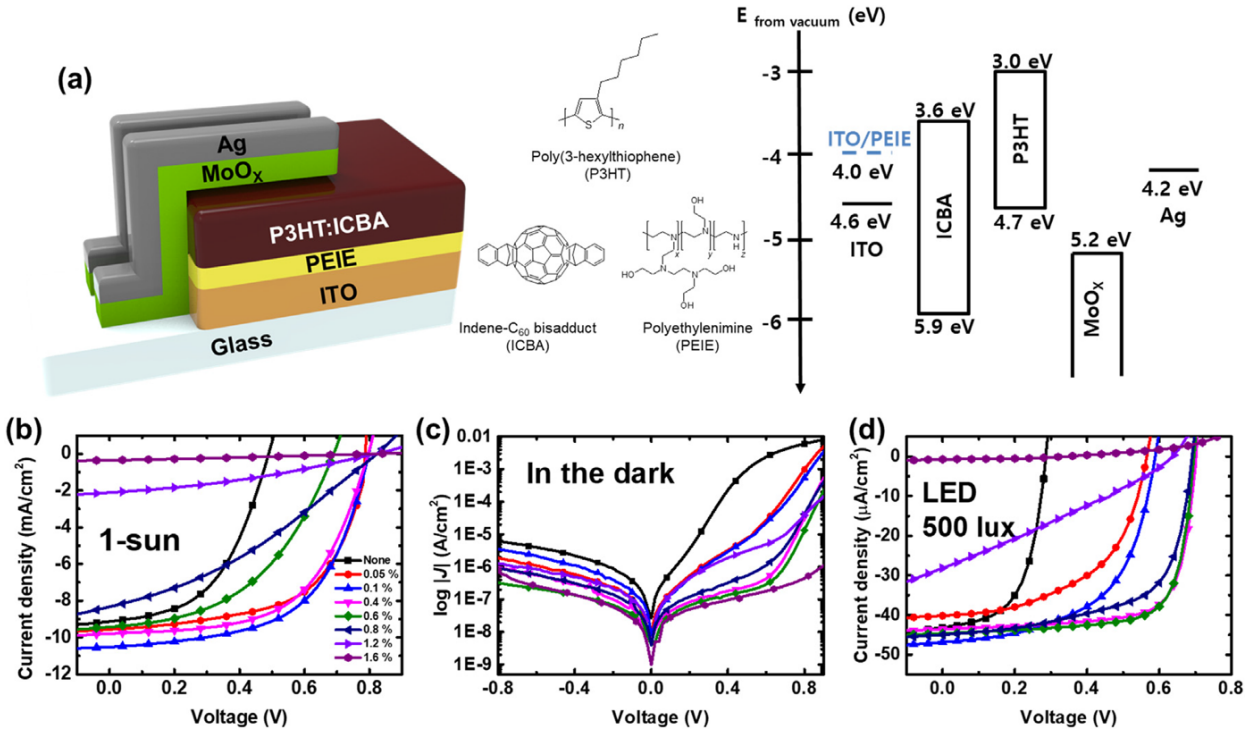

Figure 4. (a) Device structure of P3HT-ICBA-based OPV with a PEIE modified ITO electron-collecting electrode, chemical structure of P3HT, ICBA, and PEIE, and energy level diagrams of the OPV components. Representative J-V characteristics of OPVs evaluated under various light sources: (b) 1-sun illumination (100 mW/cm²), (c) in the dark, and (e) LED lamp (500 lx, $0.17 \mathrm{~mW} / \mathrm{cm}^{2}$ ). (Goo [24] 2018 Copyright Elsevier).

lating nature. Therefore, such devices showed a high $R_{P}$ of $5 \times 10^{5} \Omega \cdot \mathrm{cm}^{2}$ and PCE of $7.6 \%$ under 300-lx LED conditions, whereas the operation of thin PEIE devices was unsatisfactory (PCE of $0.7 \%$ ) because of the large leakage currents. In addition, inverted-structure OPVs with various PEIE thicknesses were reported by Goo et al. for indoor applications [24]; the device structure and characteristics are depicted in Figs. 4(a)-(d). Devices with 5.6-nm-thick-PEIE layers, which exhibited the highest PCE of $4.7 \%$ under 1-sun conditions, exhibited a PCE of $8.9 \%$ only under indoor lighting. However, devices with 8.5 $\mathrm{nm}$-thick-PEIE layers offered the highest PCE of $13.4 \%$ under 500-lx conditions, clearly indicating that a thicker PEIE layer provided low leakage current with high $R_{P}$.

In addition, Shin et al. reported the effectiveness of the ELT by comparing the properties of OPVs with various ETLs such as $\mathrm{ZnO}$ nano particles (NPs), PEIE, and ZnO NP/PEIE [25]. A single ZnO NP layer was unsuitable for indoor applications because the energy level mismatch between the ZnO NPs and ICBA induced a Schottky barrier resulting in poor performance under 500-lx LED conditions (PCE of $5.6 \%)$. The absence of ultraviolet light in an LED lamp causes an unsuitable $\mathrm{ZnO}$ NP work function (WF). However, the dipole moment caused by the deposition of the PEIE layer successfully lowered the electrode WF. Therefore, a ZnO NP/PEIE ETL effectively decreased the leakage current compared to a single ETL of ZnO NPs or PEIE. As a result, the device performance of an OPV with a $\mathrm{ZnO}$ NPs/PEIE layer was enhanced, with a PCE of $14.1 \%$ under 1000-lx LED conditions.

Furthermore, the series resistance effect for various ETLs was investigated by Shin et al. [26]. Inverted-structure OPVs based on PTB7Th: $\mathrm{PC}_{70} \mathrm{BM}$ active layers with different ETLs ( $\mathrm{PFN}, \mathrm{ZnO}, \mathrm{TiO}_{\mathrm{X}}$ ) were studied under 1-sun and 250-2000 lx LED conditions. OPVs with PFN ETL exhibited the best performance $(10.04 \%)$ under 1-sun illumination, compared to those with other layers $\left(\mathrm{TiO}_{\mathrm{X}}\right.$ and $\left.\mathrm{ZnO}\right)$; however, the worst performance was obtained under LED conditions. The energy level mismatch between the WF of the PFN and LUMO of the $\mathrm{PC}_{70} \mathrm{BM}$ caused recombination and restricted the electron selectivity of the electrode, lowering the FF and PCE. In contrast, OPVs with $\mathrm{ZnO}$ layers exhibited the best PCE (16.49\%) under LED conditions; OPVs with ZnO ETLs showed the highest photon absorption because of good agreement with AFM and transmittance. This result clearly establishes that the ETLs need to be suitably considered for design and optimization to further improve the OPV performance under indoor light conditions.

Another strategy to increase the efficiency of indoor OPVs is to enhance the light absorption. To maximize the absorption in the photoactive layer, the electrode must be designed considering the spectrum of indoor light. An oxide/metal/oxide structure can be utilized due to its electrical and optical advantages. $\mathrm{ZnO} / \mathrm{Ag} / \mathrm{ZnO}(\mathrm{ZAZ})$ electrodes are generally used as the conducting electrodes for various devices $[27,28]$. Lee et al. successfully demonstrated efficient indoor

Table 1. Photovoltaic properties of OPVs with various TCES.

\begin{tabular}{|c|c|c|c|c|c|c|c|c|}
\hline TCE & Light & $\begin{array}{c}\text { Voc } \\
(\mathrm{mV})\end{array}$ & $\begin{array}{c}J s c \\
\left(\mu \mathrm{Acm}^{-2}\right)\end{array}$ & $\begin{array}{l}\text { FF } \\
(\%)\end{array}$ & $\begin{array}{l}\text { PCE } \\
(\%)\end{array}$ & $\begin{array}{c}R_{S} \cdot \mathrm{A} \\
\left(\Omega \cdot \mathrm{cm}^{2}\right)\end{array}$ & $\begin{array}{c}R_{P} \cdot \mathrm{A} \\
\left(\Omega \cdot \mathrm{cm}^{2}\right)\end{array}$ & Ref \\
\hline Undoped $\mathrm{ZnO}$ & LED 500lx & 642 & 36.7 & 68.8 & 9.5 & 36.3 & $2.2 \times 10^{5}$ & [21] \\
\hline PEDOT: PSS & LED 500lx & 618 & 47.0 & 64.7 & 11.1 & 58 & $1.9 \times 10^{5}$ & [22] \\
\hline $\begin{array}{l}\text { PEIE modified ITO } \\
\text { (Inverted) }\end{array}$ & LED 500lx & 696 & 44.5 & 73.3 & 13.4 & 22.8 & $2.3 \times 10^{5}$ & [24] \\
\hline $\begin{array}{l}\text { ITO with } \mathrm{ZnO} \\
\text { NPs/PEIE ETL }\end{array}$ & $\begin{array}{c}\text { LED } \\
10001 \mathrm{x} \\
\end{array}$ & 713 & 85.5 & 66.8 & 14.1 & - & - & [25] \\
\hline ITO with $\mathrm{ZnO}$ ETL & $\begin{array}{c}\text { LED } \\
17501 \mathrm{x}\end{array}$ & 690 & 251.5 & 68.9 & 16.5 & 1.8 & $2.9 \times 10^{4}$ & [26] \\
\hline $\begin{array}{l}\mathrm{ZnO} / \mathrm{Ag} / \mathrm{ZnO} \\
(40 / 9 / 50 \mathrm{~nm}) \\
\end{array}$ & LED 500lx & 684 & 43.9 & 68.3 & 12.1 & 11.14 & - & [29] \\
\hline Ultrathin ITO & LED 500lx & 635 & 49.7 & 56.0 & 11.7 & 210.1 & $2.1 \times 10^{5}$ & [31] \\
\hline
\end{tabular}




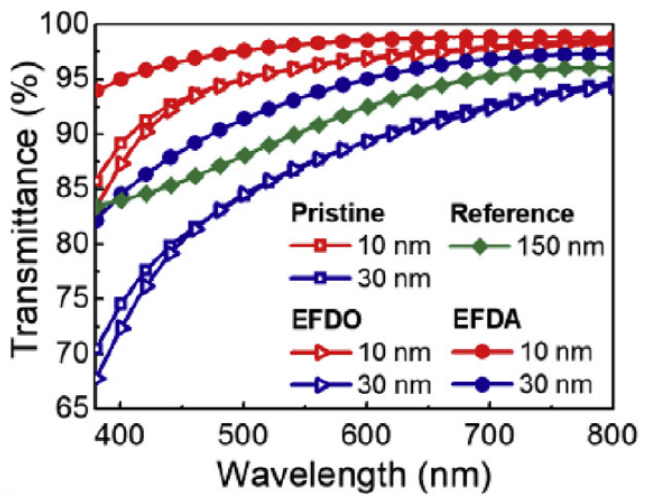

Figure 5. Transmittance of ultrathin ITO (Kim [31] 2019 Copyright Elsevier).

OPVs with ZAZ electrodes [29]. Under 1-sun illumination, the performances of OPVs with ZAZ electrodes were similar to those with ITO electrodes. However, under indoor conditions (500-lx LED), devices with ZAZ electrodes showed better power conversion efficiencies (12.3\%) than the reference devices (ITO electrodes). The microcavity effect of ZAZ electrodes enhanced the light absorption in the photoactive layer, increasing the $J_{p h}$ of OPVs [30].

Ultrathin ITO films can also be considered for application as TCEs of indoor OPVs [31]. As the transmittance and electrical conductivity are inversely proportional, sufficient film thickness is required for use as electrodes, even at the expense of the transmittance. However, the filament-doping method permits ultrathin ITO films $(10 \mathrm{~nm})$ to have enhanced conductivity, while maintaining high transmittance (as shown in Fig. 5) and low surface roughness, resulting in high performance $(11.1 \%)$ under 500 -lx LED conditions. Table 1 summarizes the overall photovoltaic properties of OPVs with various TCEs.

\section{Conclusions}

In this review, high-efficiency indoor OPVs with various types of TCEs were discussed. For low-light applications, the impact of the serial resistance is not drastic, and novel transparent electrode materials may be feasible. This property would not only contribute to the performance improvement of energy harvesting systems employing ambient energy to operate indoor electronic devices, but also lower the cost of OPVs by the usage of noble materials such as graphene or nanofibers. For outdoor applications, highly conductive electrode materials remain a requisite.

However, considerable progress needs to be made prior to largescale manufacturing of indoor OPVs. Consequently, there is a need to increase the device efficiency, improve device life, and reduce the device cost. In addition, it is important to advance module efficiencies by utilizing roll-to-roll processing or employing new designs that can reduce electrical losses. Furthermore, the OPV stability needs to be improved; this will require deeper understanding of the interactions between all the interfaces of a given cell structure, as well as improvement of both the material and device structure for oxygen and water stability, for commercial use. In order to identify materials that can achieve the above goals, large-scale production of such materials with limited batch-to-batch variation is required, and these materials need to be utilized in high-throughput processes. Currently, OPVs are applied only in niche markets that require lightweight, flexibility, and variable angle performance. However, continuous development of indoor applications will lead to the extensive application of OPV technologies in next generation devices.

\section{References}

[1] M. Freitag et al., Nat. Photonics 11, 372 (2017).

[2] H.'Aguas, T. Mateus, A. Vicente, D. Gaspar, M. J. Mendes, W. A. Schmidt, L. Pereira, E. Fortunato, and R. Martins, Adv. Funct. Mater. 25, 3592 (2015).

[3] A. Raj and D. Steingart, J. Electrochem. Soc. 165, B3130 (2018).

[4] L. C. Ding, A. Akbarzadeh, and L. Tan, Renew. Sustain. Energy Rev. 81, 799 (2018).

[5] Y. B. Lee, J. K. Han, S. Noothongkaew, S. K. Kim, W. Song, S. Myung, S. S. Lee, J. Lim, S. D. Bu, and K.S. An, Adv. Mater. 29, 1604500 (2017).

[6] M. P. Lumb et al., Adv. Energy Mater. 7, 1 (2017).

[7] Y. Zhou et al., Sci. Rep. 3, 24 (2013).

[8] Y. Aoki, Org. Electron. 48, 194 (2017).

[9] M. Freunek, M. Freunek, and L. M. Reindl, IEEE J. Photovoltaics 3, 59 (2013).

[10] B. P. Lechêne, M. Cowell, A. Pierre, J. W. Evans, P. K. Wright, and A. C. Arias, Nano Energy 26, 631 (2016).

[11] H. K. H. Lee, J. Wu, J. Barbé, S. M. Jain, S. Wood, E. M. Speller, Z. Li, F. A. Castro, J. R. Durrant, and W. C. Tsoi, J. Mater. Chem. A. 6, 5618 (2018).

[12] N. Piva, F. Greco, M. Garbugli, A. Iacchetti, V. Mattoli, and M. Caironi, Adv. Electron. Mater. 1700325 (2017).

[13] S. S. Yang, Z. C. Hsieh, M. L. Keshtov, G. D. Sharma, and F. C. Chen, Sol. RRL. 1, 1700174 (2017).

[14] C. L. Cutting, M. Bag, and D. Venkataraman, J. Mater. Chem. C. 4, 10367 (2016)

[15] C. Y. Chen, Z. H. Jian, and S. H. Huang, J. Phys. Chem. Lett. 8, 1824 (2017)

[16] A. Pivrikas, N. S. Sariciftci, G. Juška, and R. Österbacka, Res. Appl. 15, 677 (2007)

[17] S. K. Hau, H. L. Yip, and A. K. Y. Jen, Polym. Rev. 50, 474 (2010).

[18] B. Kippelen and J. L. Bredas, Energy Environ. Sci. 2, 251 (2009).

[19] R. Steim, T. Ameri, P. Schilinsky, C. Waldauf, G. Dennler, M. Scharber, and C. J. Brabec, Sol. Energy Mater. Sol. Cells 95, 3256 (2011).

[20] Y. Zhou, T. M. Khan, J. C. Liu, C. Fuentes-Hernandez, J. W. Shim, E. Najafabadi, J. P. Youngblood, R. J. Moon, and B. Kippelen, Org. Electron. 15, 661 (2014).

[21] J. S. Goo, J. H. Lee, S. C. Shin, J. S. Park, and J. W. Shim, J. Mater. Chem. A. 6, 23464 (2018).

[22] M. A. Saeed, S. H. Kim, S. Y. Lee, and J. W. Shim, Thin Solid Films 704, 138006 (2020).

[23] B. P. Lechêne, M. Cowell, A. Pierre, J.W. Evans, P. K. Wright, and A.C. Arias, Nano Energy 26, 631 (2016).

[24] J. S. Goo, S. C. Shin, Y. J. You, and J. W. Shim, Sol. Energy Mater. Sol. Cells 184, 31 (2018).

[25] S. C. Shin, Y. J., J. S. Goo, and J. W. Shim, Appl. Surf. Sci. 495, 143556 (2019).

[26] A. A. A. Torimtubun, J. G. Sánchez, J. Pallarès, and L. F. Marsal, Sustain. Energy Fuels 4, 3378 (2020).

[27] M. Girtan, Sol. Energy Mater. Sol. Cells 100, 153 (2012).

[28] J. H. Kim, J. Y. Na, S. K. Kim, Y. Z. Yoo, and T. Y. Seong, J. Electron. Mater. 44, 3967 (2015)

[29] B. R. Lee, J. S. Goo, Y. W. Kim, Y. J. You, H. Kim, S. K. Lee, J. W. Shim, and T. G. Kim, J. Power Sources 417, 61 (2019).

[30] K. D. Kim, T. Pfadler, E. Zimmermann, Y. Feng, J. A. Dorman, J. Weickert, and L. Schmidt-Mende, Apl. Mater. 3, 106105 (2015).

[31] Y. W. Kim, J. S. Goo, T. H. Lee, B. R. Lee, S. C. Shin, H. Kim, J. W. Shim, and T. G. Kim, J. Power Sources 424, 165 (2019). 